\title{
Graph Representation of the Nested Software Structure
}

\author{
Leszek Kotulski \\ Institute of Comp. Sci., Jagiellonian University, \\ Kraków, Poland \\ kotulski@ii.uj.ed.pl
}

\begin{abstract}
The use of the UML notation for software specification leads usually to lots of diagrams showing different aspects and components of the software system in a several view. Complex components are constructed by composing in parallel some elementary components and as a result the overall architecture of the system is described as a hierarchical composition of primitive components, which at the execution time may be deployed on distributed environment. The task of specifying such a system quickly becomes unmanageable without the help of some structuring tools. This paper discusses how the UML package's nested structure can be maintained and visualized with the help of a graph transformation mechanism based on the edNLC class of grammar.
\end{abstract}

\section{Introduction}

The ULM [12] is a recent approach to strengthen the effort of designing an objectoriented modeling language where all main issues of system analysis and design are taken into account. The UML provides deployment diagrams to show a system's network topology and software components that live on the network nodes. For the description of the objects replication and migration, remote interactions and dynamics network topologies, which are important issues in distributed object systems these techniques promise support, however, seems to be insufficient. Modeling distributed systems by distributed graph transformation, a powerful and flexible description, is obtained by applying graph transformation to network structures [10]. Distributed graphs are, in this case, structured with two abstraction levels: the network and the local level. The main drawback of above proposition is lack of support any structuralization mechanisms, such for example as the UML packages. In the paper we show that a graph transformation mechanism is also suitable for the visualization nested software structures. The formal background of this proposition are the ULM notation and the Rosenberg's edNLC class of grammars [5], developed next by Flasiński [2,3] and author [4,7,8].

\section{Nested Diagrams}

Deployment diagrams, used in the UML, show the configuration of run time processing elements (nodes) and software components (processes and objects) that 
execute on them. Nodes are physical processing resources, and are drawn as a 3D rectangle. Each node contains software components. The software components on different nodes can communicate across the physical connections between the nodes.

A component unit represents a modular, deployable, and replaceable part of a system that encapsulates an implementation and exposes a set of interfaces. Some components can be represented by the UML packages that provide a tool for organizing the products of analysis, design, and coding. Let us consider a management system based on a Data Warehouse concept, which stores of data obtained form an ERP systems (see Fig. 1). However, the ERP system consists from many cooperating subsystems (such as finance, sales, manufactures and human resource packages - as in fig. 2), because of that someone may want to see direct associations among these subsystems and the Data Warehouse repository. This simple example shows, that exist a necessity of introduction more than only two levels of abstraction (representing hardware or software components). That means that the method of the presentation allocated software components should create several abstraction levels (analogically as in the object oriented programming). We describe here the component and package concept using the graph transformation notation. Like a group [6] the package can be specified as a graph which visualize

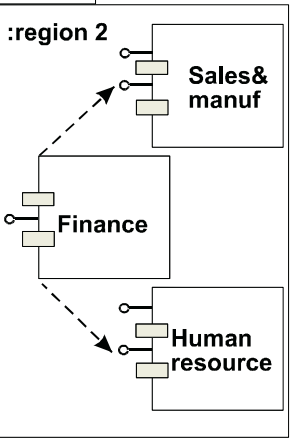

Fig. 2. connections among its components. As a result, the overall architecture of the system is described as a hierarchical composition of primitive components.

Considering visual languages both import and export interface concepts are supported [10, 11, 13]. Component diagrams in the UML knows only export interfaces, but Botch, Rumbaing and Jacobson [1] suggest that in a system consisting from a few dozen components the UML notation should directly specify imports using packages and <<import >> stereotypes. Moreover, in distributed systems the motivation of the import interface visualization is not only a possibility of formal checking the consistency of the modeled system but also the formal specification of stub instances at the implementation level.

To simplify an example we reduce components interconnection to the following: DW calls each of the ERP components to take theirs monthly summary, Finance calls appropriately Human resource and Sales for receiving daily reports and Stock (to evaluate securities). In such a case, the UML diagram presented in fig. 2 can be expressed as the attributed graph presented in fig. 3. It describes two levels of a component's configuration: the top level - representing the ERP as a final component and the internal level - representing the ERP internal structure; each component appearing inside internal structure can also has its own nesting representation; so it can create the hierarchical (tree) structure of components (where leafs are object instances). 
The presented solution is based on the node label controlled graph grammars $[2,3,4,5,7,8]$. Graph nodes used for describing of a distributed system can be labeled as follows: $\mathrm{P}$ - for a package component, $\mathrm{E}$ for an export interface, I - for an import interface (stub), $\mathrm{O}$ - for an object instance implementing component, $\mathrm{N}$ - for a computing node representation. All additional information about the graph node can be described by its attributes. Graphically we expose only component's name. The only correct labeling pattern $^{1}$ is included is the following set of triples $(\mathrm{E}, \mathrm{b}, \mathrm{P}),(\mathrm{E}, \mathrm{b}, \mathrm{O}),(\mathrm{P}, \mathrm{c}, \mathrm{I}),(\mathrm{O}, \mathrm{b}, \mathrm{G}),(\mathrm{O}, \mathrm{c}, \mathrm{I}),(\mathrm{N}, \mathrm{d}, \mathrm{G})$, $(\mathrm{N}, \mathrm{d}, \mathrm{O}),(\mathrm{I}, \mathrm{l}, \mathrm{E}),(\mathrm{N}, \mathrm{n}, \mathrm{N})$. Edge labels b, c, d, 1 and $\mathrm{n}$ are appropriately abbreviation for belonging, calls, deploy, linked and node interconnection.

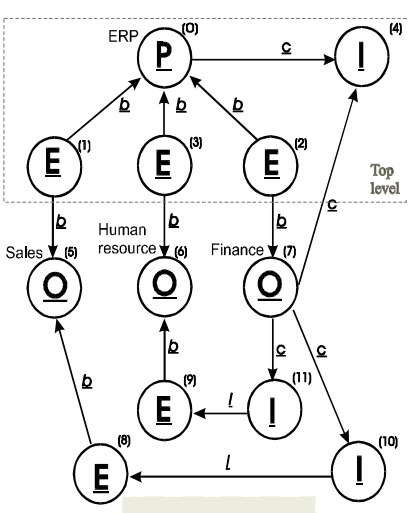

Fig. 3.

The presented transformation from the UML to the graph notation seems to be simple and intuitive. On the other hand, when size both package and allocation graphs grows to hundreds or thousands nodes we should also consider a computational complexity of the considered solution. Let us notice that the membership problem i.e the parsing of the graph in order to designate the proper sequence of productions has the critical complexity for many graph grammars (among others for graph transformation model based on graph morphism [10,11]). For this reason we suggest using of the node label controlled class of graph grammars. The introduced in $[7,8]$ aedNLC graph grammar (as equivalent to ETPL(k) graph grammar [3]) allows one to solve parsing, membership and a new graph generation problems with $\mathrm{O}\left(\mathrm{n}^{2}\right)$ computational complexity.

The correctness of graph transformation is forced by a graph transformation rules called productions described how the left hand production graph be replaced by the right hand one with the embedding transformation defining how edges coming to/from the left hand side graph be connected with the right hand one. For example if we would like to express that some service can be offered by an object we use the following production with the embedding transformation

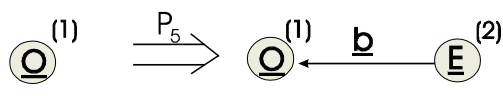
$\mathbb{E}_{5}=\{(($ op,out, 1$), \quad\{(\mathrm{O},(\mathrm{P}$, true $)$, op, out $),(\mathrm{E},(\mathrm{P}$, true $)$, pe, in $)\})$, COPY_REST $\}$ interpreted as follows every edge labeled by "op" and coming from the removed node in the visualization graph ought to be replaced by the edge (labeled by op) connecting the node of the graph of the righ -hand side of the production and labeled by "O" with the node $p$ of the rest graph and labeled by "P" and the edge (labeled by pe) connecting the node of the graph of the righ -hand side of the production and labeled by "E" with the node $p$ of the rest graph and labeled by "P". Note that edges mentioned in the embedding transformation are the auxiliary ones and are not expressed in the graph presented in fig. 3. The full description of the set of the productions used for creation of the visualization graph is presented in the technical raport [8].

${ }^{1}$ Term labeling pattern for edge $(\mathrm{v}, \mu, \mathrm{w})$ means triple $(\delta(\mathrm{v}), \mu, \delta(\mathrm{w}))$, where $\delta(\mathrm{v})$ and $\delta(\mathrm{w})$ returns labels of $\mathrm{v}, \mathrm{w}$ node indices appropriately. 


\section{Conclusions}

In the previous chapter it is shown that the graph transformation can be useful formalism for controlling the allocation process. The deployed software component can be split onto a few abstraction levels using packages. It improve a quality of a distributed system presentation; now we can show either a nested structure of a component or an allocation graph in a flat form (where any component is either in their top or internal level of specification; two different, non nested components can be, however, described at the different abstraction level [8)]. Moreover, as the package is specified by the attributed graph, so it can formally check the correctness of its component deployment and the consistency of the generated software (i.e. assure that for each request a proper service is associated). As it shown in [7,8] the aedNLC grammars allows one to divide such an allocation graph onto a few distributed subgraphs and control their modification in a parallel way. The last property is important not only from the computational complexity point of view but also creates the practical possibility of a parallel work of several systems administrators.

\section{References}

1. G. Booch, J. Rumbaugh, I. Jacobson: The Unified Modeling Language - User Guide. Addison Wesley Longman, Inc. 1999.

2. Flasiński M.: Characteristic of edNLC-graph Grammars for Syntactic Pattern Recognition. Computer Vision, Graphics and Image Processing, 1989, vol.42, pp. 1-21.

3. Flasiński M.: Power Properties of NCL Graph Grammars with a Polynomial Membership Problem. Theoretical Computer Science, 1998, vol. 201, pp. 189-231.

4. Flasiński M., Kotulski L.: On the Use of Graph Grammars for the Control of a Distributed Software Allocation. The Computer Journal, 1992, vol. 35, pp. A167-A175.

5. Janssens D., Rozenberg G., Verraedt R.: On Sequential and Parallel Node-rewriting Graph Grammars. Computer Graphics and Image Processing, 1982, vol. 18, pp. 279-304.

6. Kotulski L., Jurek J., Moczurad W.: Object-Oriented Programming in the Large Using Group Concept. Computer Systems and Software Engineering - 6th Annual European Conference, Hague 1992, pp. 510-514.

7. Kotulski L.: Model systemu wspomagania generacji oprogramowania współbieżnego w środowisku rozproszonym za pomocą gramatyk grafowych. Postdoctorals Lecturing Qualifications. Jagiellonian University Press, ISBN 83-233-1391-1, 2000.

8. Kotulski L.: Parallel Allocation of the Distributed Software Using Node Label Controlled Graph Grammars. Jagiellonian University, Inst. of Comp. Sci., Preprint no. 2003/006

9. Kotulski L.: Nested Software Structure generated by aedNLC graph grammar - technical report. Jagiellonian University, Inst. of Comp. Sci., Preprint no. 2005/003 http://www.ii.uj.edu.pl/preprint/kotulski03

10. Taentzer G., Fischer I., Koch M., Vole V.: Visual design of distributed graph transformation. In Handbook of Graph Grammars and Computing by Graph Transformation, Volume 3: Concurrency, Parallelism, and Distribution. World Scientific, 1999.

11. Taentzer G.: A Visual Modeling Framework for Distributed Object Computing. In Formal methods for open object-based distributed systems, Kluwer Academic Publishers, 2002

12. OMG-Unified Modeling Language, v1,5. www.rational.com

13. Zhang D., Zhang K., Cao J.: A context-sensitive Graph Grammar Formalism for the Specification of Visual Languages. The Computer Journal, 2001, vol 44, no. 4, pp186-200. 